\title{
Sobre las nuevas relaciones entre Gravedad y la Teoría Cuántica de Campos
}

\section{On the new relations between Gravity and Quantum Field Theories}

\author{
BRYAN LARIOS ${ }^{1}$
}

Recibido: 3 de mayo de 2019/ Aceptado: 10 de Junio de 2019

\begin{abstract}
En este trabajo se comentan los aspectos modernos de la teoría cuántica de campos y su conexión con la teoría de gravedad. Por más de 60 años se han tratado los campos cuánticos como los bloques fundamentales en física de altas energías. De igual manera, en la relatividad general de Einstein se ha trabajado por casi 100 años con la métrica del espaciotiempo como lo primordial. Veremos que si cambiamos de paradigmas y consideramos los observables físicos como lo fundamental, las dos teorías pilares de la física presentan sorprendentes y modernas relaciones matemáticas, las cuales son el actual campo de investigación en la física fundamental de vanguardia.
\end{abstract}

In this work, we review the modern aspects of quantum field theory and its relations with the theory of gravity. Around 60 years, the quantum fields has been the fundamental blocks in high energy physics. Similarly, for more than 100 years in the Einstein theory of general relativity, it has been the metric field of space-time the primordial. We shall see that changing our paradigms and considering the physical observables as fundamental, the two pillars of physics reveal amazing mathematical relations.

\section{PALABRAS CLAVES}

campos cuánticos, amplitudes, gravedad, doble copia

\section{KEYWORDS}

quantum fields, amplitudes, gravity, double copy

PACS

03.70.+k,11.10.-z
${ }^{1}$ Escuela de Física, Facultad de Ciencias, Universidad Nacional Autónoma de Honduras email:bryanlarios@gmail.com

\begin{abstract}
Agradecimientos
Agradezco al Jefe del Departamento de Gravitación y Altas Energías de la Escuela de Física de la UNAH por permitirme realizar este trabajo. Agradezco a mis colaboradores de la Benemérita Universidad Autónoma de Puebla. Sobre todo, agradezco a tres de mis alumnos del curso de Tópicos de Física Teórica, Juan José, Selvin Roberto y Daniel Amaya, por ayudarme a mantener vivo el espíritu de la verdadera academia.
\end{abstract}

\section{I | INTRODUCCIÓN}

L a teoría de la relatividad general de Einstein (GR por sus siglas en Inglés) unifica la gravedad y la geometría. Esta monumental teoría ha permitido estudiar y describir por más de 100 años una gran variedad de fenómenos clásicos en nuestro Universo (Carroll, 2004). No hay ninguna duda en la comunidad científica que GR describe la dinámica del Cosmos a gran escala. Por otro lado, a escalas microscópicas, el mundo se ve gobernado por el carácter probabilístico y no es posible describir a esta escala ningún fenómeno con conceptos o herramientas puramente clásicas como por ejemplo; la teoría 
de la relatividad general y las ecuaciones de Maxwell (Weinberg, 2013). Justo antes del año milagroso del aún joven Albert Einstein, el físico alemán Max Plank concibió la idea de un flujo de energía discretizado, o bien como le conocemos ahora, "cuantizado" (Weinberg, 2003). La física cuántica desde su génesis ha tenido un tremendo impacto y éxito en la comunidad científica en general. La versión más moderna y precisa que describe no solo los fenómenos microscópicos, sino también los de altas energías (velocidades cercanas a la velocidad de la luz, $c=3.0 \times{ }^{8} \mathrm{~m} / \mathrm{s}$ ) es conocida como la Teoría Cuántica de Campos, o simplemente QFT por sus siglas en inglés.

Los conceptos fundamentales que subyacen en los cálculos de extrema precision que requiere el LHC (Large Hadron Collider por sus siglas en inglés) en el CERN (European Organization for Nuclear Research por sus siglas en inglés) se sustentan en QFT. Dicho de otra forma, QFT es la base del modelo estándar de partículas elementales (SM por sus siglas en inglés), donde se unifican la interacción fuerte, débil y electromagnética, pero no así la interacción gravitacional (Zwiebach, 2004). Es importante aclarar que QFT es una plataforma general, el SM es apenas una teoría cuántica de campos con números cuánticos en específico (Weinberg, 1996). En principio existen infinitas QFTs; por el momento, el SM es la que mejor describe nuestro Universo a escala microscópica (Weinberg, 1996), (Weinberg, 1967). La validez de QFT no solo es en el régimen de altas energías; en un caso efectivo (bajas energías) es igual una herramienta poderosa para física de materia condensada, sobre todo para entender el problema de muchos cuerpos cuánticos que surge en la teoría de metales; de igual forma para el estudio de superconductividad, el efecto Hall cuántico entre muchos otros que no discutiremos en este trabajo (Lancaster \& Blundell, 2014).

A pesar de que por un siglo hemos tenido control de la física que describe la dinámica de los cuerpos supermasivos a escalas cósmicas, y de igual manera, aunque de forma separada contamos con una descripción aun más precisa para el estudio de las interacciones a escalas microcópicas, muchos fenómenos naturales continuan sin ser explicados (Cottingham \& Greenwood, 2007), (Larios, 2017), (Martin, 1998). Ese es el caso de los efectos de la gravedad a escalas del orden de $10^{-15} \mathrm{~m}-10^{-35} \mathrm{~m}$, es decir los efectos cuánticos de la gravedad (Hawking, 1975). Otro paradigma abierto en la física fundamental es determinar cuál es la naturaleza de la materia y energía oscura (Diaz-Cruz \& Larios, 2016). Existen por supuesto además de estos grandes enigmas de la física, muchos más, pero quizás ya no tan fundamentales. En este trabajo nos enfocaremos únicamente en los aspectos cuánticos de la gravedad.

En los últimos 15 años, la física fundamental ha logrado acumular múltiples y constantes triunfos que convergen a un monumental avance, tal es el caso que incluso algunos pilares de la física podrían ser desplazados por nuevos fundamentos (Nima Arkani-Hamed, 2010). Quizás estemos viviendo una época parecida a la de James Clerk Maxwell, donde se establece por primera vez la relación formal entre el campo eléctrico y el magnético, o incluso la época del ya maduro Albert Einstein, donde se unifica la gravedad con la geometría (Landau \& Lifshitz, 1975). Las diferentes relaciones que han surgido (y continúan surgiendo) entre teorías de gravedad y algunas teorías cuánticas son ejemplo claro de la revolución paradigmática que se vive y disfruta en la física y matemática de frontera (H. Kawai. 1985), (Witten 2004), (Z. Bern, 2008).

El trabajo tiene la siguiente organización; en la Sección [ se plantea el estado del arte del SM y GR, y se menciona la incompatibilidad de estas teorías a nivel de los Lagrangianos. Posteriormente en la Sección III se introduce la teoría clásica de campos y la cuantización canónica. En la Sección III] se introducen los conceptos de observables físicos y la importancia que tienen debido a que conectan 
las mediciones experimentales con las predicciones teóricas. En esta misma sección, se introduce la amplitud de probabilidad como el bloque fundamental que sirve para calcular los observables físicos. En la Sección IV], hacemos un intervalo para mencionar brevemente la importancia de los métodos perturbativos en QFT, de igual manera se plantean las preguntas fundamentales de este trabajo. En la Sección V] se introduce el Lagrangiano de Hilbert-Einstein, mismo que servirá para contrastar con el Lagrangiano (una parte) del SM. También se introduce en esta misma sección la doble copia, donde la gravedad se expresa perturbativamente como el cuadrado de una teoría cuántica. Finalmente, en la Sección VI] se dan las conclusiones de este trabajo.

\section{II | INTRODUCCIÓN A LA TEORÍA CUÁNTICA DE CAMPOS}

En esta sección se introduce muy brevemente la cuantización canónica (Weinberg, 1996), la cual tiene como punto de partida una teoría clásica de campos, como la electrodinámica y GR. Básicamente se parte de un Lagrangiano (o densidad Lagrangiana) con campos clásicos que respetan los corchetes de Poisson (Peskin \& Schroeder, 1995). Se promueven dichos campos clásicos a operadores que respeten un álgebra de conmutadores, también conocida como álgebra de Dirac (Weinberg, 1996), (Peskin \& Schroeder, 1995). Dicho procedimiento es conocido como cuantización canónica, y una vez realizado se dice que la teoría ha sido cuantizada, y ahora está lista para realizar cálculos (generalmente perturbativos) que sirven para obtener predicciones teóricas de observables físicos a nivel cuántico.

\section{I Cuantización Canónica (método tradicional)}

La teoría clásica de campos se encarga de describir la dinámica de un sistema clásico, como por ejemplo la dinámica de los campos electromagnéticos $(\vec{E}$ y $\vec{B})$ (Jackson, 1998). Es de gran interés a este nivel, que la teoría sea manifiestamente invariante bajo transformaciones de Lorentz, para que respete los postulados de la relatividad especial (Landau \& Lifshitz, 1975). Una vez conozcamos el Lagrangiano covariante de la teoría clásica de campos (básicamente un Lagrangiano invariante bajo transformaciones de Lorentz y además hermítico) se calculan las ecuaciones de movimiento utilizando el principio de Hamilton o bien directamente las ecuaciones de Euler-Lagrange (Weinberg, 1996). Para ilustrar mostramos el caso de la densidad Lagrangiana de Maxwell, el cual toma la siguiente forma (Jackson, 1998):

$$
\mathcal{L}=-\frac{1}{4} F^{\mu v} F_{\mu v}-A^{\mu} J_{\mu}
$$

cuyas ecuaciones de movimiento (después de utilizar Euler-Lagrange, $\partial_{\alpha}\left(\frac{\partial \mathcal{L}}{\partial_{\alpha} A^{\mu}}\right)-\frac{\partial \mathcal{L}}{\partial A^{\mu}}=0$ ) son las siguientes:

$$
\begin{aligned}
\partial_{\mu} F^{\mu \nu} & =J^{v}, \quad \text { Ecuaciones de Maxwell con fuente. } \\
\partial^{\alpha} F^{\mu \nu}+\partial^{\mu} F^{v \alpha}+\partial^{v} F^{\alpha \mu} & =0, \quad \text { Ecuaciones de Maxwell sin fuente. }
\end{aligned}
$$

donde $F^{\mu v}$ es el tensor de Faraday, y está definido como sigue (Jackson, 1998):

$$
F^{\mu v}=\partial^{\mu} A^{v}-\partial^{v} A^{\mu}
$$

El cuadrivector $A^{\mu}(t, \vec{x})=(\phi(t, \vec{x}), \vec{A}(t, \vec{x}))$ es una manifestación directa de la unificación del espaciotiempo, conocido en este caso como el espacio de Minkowski (Weinberg, 1996), y contiene en su componente temporal al potencial escalar $\phi(t, \vec{x})$ y en sus componentes espaciales $\left(\mathbb{R}^{3}\right)$ al potencial vectorial $\vec{A}(t, \vec{x})$. Como es bien conocido, el campo magnético y el eléctrico están definidos en términos 
de estos potenciales de la siguiente forma:

$$
\begin{aligned}
\vec{E}(t, \vec{x}) & =-\vec{\nabla} \phi(t, \vec{x})-\frac{\partial \vec{A}(t, \vec{x})}{\partial t}, \\
\vec{B}(t, \vec{x}) & =\vec{\nabla} \times \vec{A}(t, \vec{x}) .
\end{aligned}
$$

A nivel cuántico, se verá que los campos $\vec{E}$ y $\vec{B}$ describen al fotón. Por otro lado, ¿qué mecanismo genera fotones a nivel cuántico?, ¿quien es su fuente? En la ecuación covariante de Maxwell con fuente, eq. (2), vemos que aparece un término tipo energía potencial, o como lo llamaremos de ahora en adelante "término de interacción", es decir $A^{\mu} J_{\mu}$ describe al campo electromagnético $A^{\mu}$ interactuando con su fuente $J^{\mu}((t, \vec{x}))=(\rho(t, \vec{x}), \vec{J}(t, \vec{x}))$, donde $\rho(t, \vec{x})$ es la densidad de carga y $\vec{J}(t, \vec{x})$ es la densidad de corriente, ambas cantidades conservadas $\left(\partial_{\mu} J^{\mu}=\frac{\partial \rho}{\partial t}+\vec{\nabla} \cdot \vec{J}=0\right)$. Resulta interesante que a nivel cuántico la fuente no es más que la interacción entre fermiones, por ejemplo un electrón absorbe un fotón y emite un positrón, o bien un electrón colisiona con un positrón se aniquilan y generan luz a nivel cuántico, es decir, se emiten fotones.

Como mencionamos anteriormente, los campos clásicos respetan los corchetes de Poisson (Weinberg. 1996); para el caso de la electrodinámica toma la siguiente forma

$$
\left\{A^{\mu}(t, \vec{x}), \dot{A}^{v}(t, \vec{y})\right\}=-\delta^{\mu v} \delta^{3}(\vec{x}-\vec{y}),
$$

donde $\delta_{\mu v}$ es la Delta de Kronecker para el espacio de Minkowski (o de la variedad Lorenziana).

\section{2 | Regla de correspondencia}

Una vez tenemos el corchete de Poisson de los campos clásicos (y su momento conjugado $\Pi^{\mu}(t, \vec{x})=$ $\frac{\partial \mathcal{L}}{\partial \dot{A}_{\mu}}$ ), podemos decir que estamos a un paso para cuantizar canónicamente (Weinberg, 1996), (Peskin \& Schroeder, 1995). Paul Dirac utilizando su método de constricciones (Dirac, 1958) encontró que para cuantizar una teoría clásica de forma canónica se debe de imponer la siguiente correspondencia:

$$
\{A(t, \vec{x}), B(t, \vec{y})\} \rightarrow \frac{1}{i \hbar}[\hat{A}(t, \vec{x}), \hat{B}(t, \vec{y})]
$$

donde $A(t, \vec{x})$ y $B(t, \vec{x})$ son dos variables canónicamente conjugadas. Aplicando la correspondencia de la eq. (8) al corchete de Poisson de la eq. (7), obtenemos la siguiente relación de conmutación:

$$
\left[\hat{A}^{\mu}(t, \vec{x}), \dot{\hat{A}}^{v}(t, \vec{y})\right]=i \delta^{\mu v} \delta^{3}(\vec{x}-\vec{y}), \quad \hbar=1 .
$$

Promoviendo la solución más general posible de las ecuaciones de Maxwell a operador cuántico, tenemos:

$$
\hat{A}^{\mu}(t, \vec{y})=\sum_{\lambda=0}^{3} \int_{-\infty}^{\infty} \frac{d k^{3}}{\sqrt{(2 \pi)^{3}}} \frac{1}{\sqrt{2 \omega_{k}}}\left(e^{-i k^{\mu} x_{\mu}} \varepsilon_{\lambda}^{\mu}(\vec{k}) \hat{a}_{k}^{\lambda}+e^{i k^{\mu} x_{\mu}} \varepsilon_{\lambda}^{* \mu}(\vec{k}) \hat{a}_{k}^{\dagger \lambda}\right),
$$

donde $\omega_{k}$ es la frecuencia angular relacionada con la energía $\left(E=\hbar \omega_{k}\right)$ y con el momento $\left(\omega_{k}=k c\right)$. Luego los cuadrivectores $x^{\mu}$ y $k^{\mu}$ definen la cuadriposición y el cuadrimomento, respectivamente, es decir $x^{\mu}=(t, \vec{x})$ y $k^{\mu}=\left(\omega_{k}, \vec{k}\right)$. Luego se tiene el cuadrivector de polarización $\varepsilon_{\lambda}^{\mu}(\vec{k})$, y finalmente $\hat{a}_{k}^{\lambda}$, que son los coeficientes de Fourier que heredan el carácter de operador del campo. Sustituyendo la eq. (10) en la eq. 9], obtenemos el álgebra de conmutación para los operadores escalera que nos 
servirán para crear y destruir partículas; esto es como sigue:

$$
\left[\hat{a}_{k}^{\lambda}, \hat{a}_{k^{\prime}}^{\dagger \lambda^{\prime}}\right]=-\eta^{\lambda \lambda^{\prime}} \delta^{3}\left(\vec{k}-\vec{k}^{\prime}\right)
$$

Ahora tenemos las reglas que regulan los operadores escalera, es decir conocemos su álgebra de conmutadores, y en consecuencia tenemos control sobre cómo crear y destruir partículas (Weinberg. 1996). Por ejemplo un estado cuántico con un fotón con momento $\vec{k}_{1}$ se describe $\left|k_{1}\right\rangle=\hat{a}_{k_{1}}^{\dagger \lambda}|0\rangle$. De mecánica cuántica sabemos que $|0\rangle$ es el estado de menor energía (el vacío), y en este caso $\lambda$ define los grados de libertad del vector de polarización; es decir, si este es transversal o longitudinal, en caso de vectores masivos.

\section{OBSERVABLES FÍSICOS (NIVEL CUÁNTICO)}

En esta sección nos enfocamos en los observables físicos, es decir en las cantidades que pueden ser corroboradas experimentalmente. A nivel cuántico, los observables físicos son la sección diferencial de corte $\left(\frac{d \sigma}{d \omega}\right)$ y el tiempo de vida medio de una partícula $(\tau)$ Ambas cantidades son el tema central de los aceleradores de partículas, como el LHC en el CERN. Existe una gran cantidad de experimentos, como los de expectrometría y difracción de rayos X que se basan en la sección diferencial de corte (para las aplicaciones de QFT, ver (Lancaster \& Blundell, 2014)). Dado que este trabajo tiene interés puramente formal, nos interesa conocer cuál es la relación entre estos dos observables y nuestra teoría ahora cuantizada canónicamente.

\section{I Amplitud de Probabilidad}

Los estados cuánticos evolucionan temporalmente, tienen una dinámica. En el caso de una teoría no relativista (bajas energías) y de partículas con espín 0, la dinámica del estado la gobierna la ecuación de Schrodinger $\left(\hat{H}_{S}|\psi(t)\rangle=i \hbar \frac{\partial}{\partial t}|\psi(t)\rangle\right)$. [cite]. En sistemas cuánticos interactuantes, nos interesa conocer la probabilidad de transición de un estado inicial $|i\rangle$ (sin interactuar o libre) a un estado final $|f\rangle$ (después de interactuar). Justamente la amplitud de probabilidad nos permite conocer esta evolución temporal; esta cantidad física puramente cuántica (debido a su carácter probabilístico) se define como sigue:

$$
\mathcal{A}=\langle f|\hat{S}| i\rangle=S_{f i},
$$

El operador $S$ es conocido como la matriz de transición $S$ y se calcula a partir del Hamiltoniano de interacción (potencial) (Weinberg, 1996). Los observables físicos que se miden de forma experimental en los aceleradores de partículas como el LHC en el CERN, son la sección diferencial de corte y el tiempo de vida media. Es justamente QFT quien nos permite conectar las predicciones teóricas con la enorme cantidad de datos que obtienen los detectores de partículas. Estas cantidades están definidas como sigue:

$$
\begin{aligned}
\frac{d \sigma}{d \Omega} & \propto|\mathcal{A}|^{2}, \\
\tau & \propto \frac{1}{|\mathcal{A}|^{2}},
\end{aligned}
$$

Notar que la amplitud de probabilidad $\mathcal{A}$ pertenece a los números complejos. La amplitud $\mathcal{A}$ que se calcula con métodos perturbativos de QFT conecta la teoría con el experimento [cite]. 


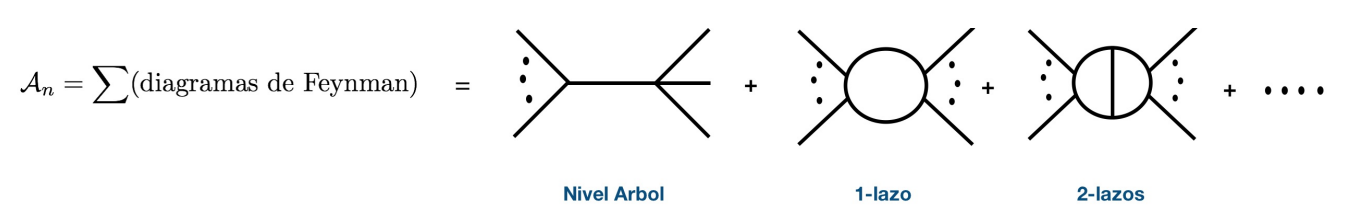

Figure 1: Amplitud de Probabilidad a partir de diagramas de Feynman

\section{2 | Calculando Amplitudes con el método de Feynman}

La amplitud de probabilidad nos dice qué tan probable es que un sistema evolucione de un estado inicial a uno final cuando este es interactuante. Richard Feynman encontró un método para calcular la amplitud $\mathcal{A}$ de cualquier interacción a partir de una serie de diagramas, conocidos en su honor como diagramas de Feynman. Feynman nos enseñó a construir la amplitud a partir de los diagramas que se dibujan de manera muy intuitiva, los cuales se deducen a partir de la densidad Lagrangiana (Weinberg, 1996). De lo contrario, existe el método ideado por Julian Schwinger (Schwinger, 1951) que requiere mucho más trabajo y es menos intuitivo. En los diagramas de la Figura (1) se muestra un diagrama a nivel árbol (primer orden perturbativo) y los demás a nivel de lazos (orden mayor en la expansión perturbativa). Cada vértice de los diagramas y cada línea (interna o bien externa) representa una expresión matemática que finalmente permiten construir la amplitud de probabilidad de un proceso físico en específico. En gran parte, el modelo estándar de partículas elementales es una especie de receta que nos permite realizar cálculos con el método de Feynman (Cottingham \& Greenwood 2007), (Peskin \& Schroeder, 1995).

\section{IV | MÉTODOS PERTURBATIVOS MODERNOS “INTERLUDIO”}

Para describir la dinámica de un sistema interactuante a nivel cuántico, es necesario resolver las ecuaciones de movimiento. Resulta que las ecuaciones diferencias parciales (PDF por sus siglas en inglés) que describen la dinámica de los campos no son lineales, por lo que para resolverlas se recurre a métodos perturbativos (Peskin \& Schroeder, 1995). Por ejemplo, si tenemos una teoría descrita por un campo escalar real $\phi(t, \vec{x})$ con un potencial (término de interacción) de la forma $V(\phi(t, \vec{x}))=\frac{\lambda}{3 !} \phi(t, \vec{x})^{3}$, el Lagrangiano es el siguiente (Peskin \& Schroeder, 1995)

$$
\mathcal{L}=\frac{1}{2} \partial_{\mu} \phi \partial^{\mu} \phi-V(\phi)=\frac{1}{2}\left(\left(\frac{\partial \phi}{\partial t}\right)^{2}-(\vec{\nabla} \phi)^{2}\right)-\frac{\lambda}{3 !} \phi(t, \vec{x})^{3}
$$

cuya ecuación de movimiento toma la siguiente forma

$$
\begin{aligned}
\partial_{\mu} \partial^{\mu} \phi+\lambda \phi^{2}=0, & \text { forma covariante } \\
\square \phi+\lambda \phi^{2}=0, & \text { forma estándar. }
\end{aligned}
$$

Se puede notar en la ecuación de movimiento (17) la no linealidad de la PDF. Este tipo de ecuaciones de movimiento no se puede resolver de forma analítica y exacta; es necesario recurrir a métodos perturbativos como los mencionados en la Sección 1

En esta sección se introduce de manera muy pragmática algunas de las nuevas técnicas perturbativas de QFT; ver la siguiente referencia para más detalles (O. M.-A. J. Lorenzo Diaz-Cruz Bryan O. Larios, 2016). En el año 2003 Edward Witten (Witten, 2004) encuentra una conexión entre las teorías de norma y los twistores de Penrose (Penrose \& MacCallum 1972), lo que revoluciona increíblemente 
nuestra visión de QFT.

Tradicionalmente en física de altas energías se ha recurrido a la formulación Lagrangiana que depende de los campos para poder construir las amplitudes $\mathcal{A}$, las cuales como se mencionó, son el corazón de QFT ya que conectan el experimento con la teoría (Peskin \& Schroeder, 1995). En ese sentido, la pregunta que surge naturalmente es la siguiente; ¿Son realmente los campos cuánticos fundamentales o debemos cambiar de paradigma?

\section{V | REINTERPRETANDO LA GRAVEDAD}

En esta sección comenzamos haciendo un recuento de los principales resultados en GR. Posteriormente introducimos la doble copia, donde la gravedad se expresa como el cuadrado de una teoría cuántica (Z. Bern, 2008).

\section{I Lagrangiano de Relatividad General (gravedad)}

Las ecuaciones de campo de Albert Einstein se dedujeron inicialmente de manera muy intuitiva (Carroll. 2004), aunque posteriormente con el aporte del matemático David Hilbert se formúló una densidad Lagrangiana que al variarla con respecto a la métrica reproduce las mismas ecuaciones de campo. La acción que incluye la densidad Lagrangiano de Einstein-Hilbert es la siguiente (Carroll, 2004)

$$
S_{H E}=\frac{1}{k^{2}} \int \sqrt{-g} R d^{4} x
$$

donde $R$ es el escalar de Ricci y $g$ es el determinante de la métrica del espacio-tiempo curvo, es decir $g=\operatorname{det}\left(g_{\mu v}\right)$. Al variar la acción (18) con respecto a la métrica $\left(\delta S_{H E} / \delta g_{\mu v}=0\right)$ encontramos la siguiente ecuación de movimiento:

$$
R_{\mu v}-g_{\mu v} R=8 \pi G T_{\mu v},
$$

donde $R_{\mu v}$ es el tensor de Ricci y $T_{\mu v}$ es el tensor de energía momento. La ecuación de movimiento 19] es la famosa ecuación de campo de Einstein, y el lado izquierdo nos dice cómo el espacio-tiempo se curva, mientras que el lado derecho de la ecuación codifica la materia que genera dicha curvatura (Carroll 2004). La ecuación (19) en el límite de un campo $g_{\mu \nu}(t, \vec{x})$ débil reproduce la ecuación de campo de Newton $\nabla^{2} \Phi(x)=4 \pi G \rho($ Carroll, 2004).

\section{2 | Gravedad como doble copia de una teoría cuántica}

El modelo estándar de partículas elementales (SM), es una teoría cuántica de campos que unifica las interacciones débil, fuerte y la electromagnética (Peskin \& Schroeder, 1995), (Cottingham \& Greenwood, 2007). El SM no incluye la gravedad y en ese sentido tiene que ser un modelo efectivo de una teoría más general (Zwiebach, 2004) que sí involucre las cuatro interacciones fundamentales de la naturaleza conocidas hasta ahora. A pesar de las notables diferencias entre la teoría de la gravedad y el SM, las que se pueden apreciar fundamentalmente en sus respectivos Lagrangianos (Eq. (1) $]^{1} \mathrm{y}$ (18)). Recientemente se ha encontrado que si renunciamos a los campos cuánticos como bloques fundamentales y más bien vemos a las amplitudes $\mathcal{A}$ como los principales objetos microscópicos,

\footnotetext{
1 Esta densidad Lagrangiana describe únicamente al campo electromagnético, sin embargo la Lagrangiana de la interacción fuerte que describe los gluones es muy similar. De igual forma, la Lagrangiana que describe los bosones de norma masivos Peskin \& Schroeder. 1995.
} 


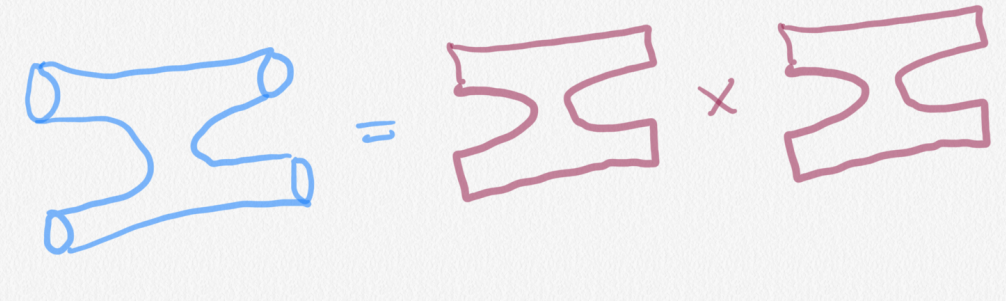

Figure 2: La amplitud de 4 cuerdas cerradas es igual a una doble copia de la amplitud de 4 cuerdas abiertas.

surgen relaciones matemáticas increíbles que permiten ver la gravedad como una doble copia de la teoría cuántica (Z. Bern, 2008), (H. Kawai, 1985), es decir

$$
\text { Gravedad }=\text { Teoría Cuántica } \times \text { Teoría Cuántica }
$$

La relación matemática de la ec. (20) nos dice que a nivel perturbativo la amplitud de gravitones $\mathcal{A}_{\text {gravitones }}{ }^{2}$ es igual a una doble copia de amplitudes de gluones $\left(\mathrm{Z}\right.$. Bern, 2008) $\left(\mathcal{A}_{Y M}\right)$. Incluso se puede dar con una amplitud que involucre fotones, cuando aparecen acoplados a campos masivos (M. A. J. Lorenzo Diaz-Cruz Bryan Larios, d). La relación matemática toma la siguiente forma

$$
\mathcal{A}_{\text {gravitones }}=\mathcal{A}_{Y M} \times \mathcal{A}_{Y M} \text {. }
$$

El resultado de la ec. 21) nos permite hacer cálculos que antes eran imposibles de realizar con los métodos perturbativos tradicionales (ver la referencia (DeWitt, 1967) para ver un ejemplo de amplitudes de gravitones con los métodos perturbativos tradicionales). Gracias al tremendo avance que se ha alcanzado respecto a comprender (o quizás cambiar) los fundamentos de QFT y gravedad, solo es necesario evaluar la amplitud en el lado cúantico $\left(\mathcal{A}_{Y M}\right)$ y luego elevarla al cuadrado para obtener el resultado del lado de la gravedad. Este tipo de relaciones "Gravedad $\sim$ Cuántica" " solo tiene precedentes en la teoría de cuerdas, donde una amplitud con cuatro cuerdas cerradas (las cuerdas cerradas corresponden a gravitones) es igual al cuadrado de amplitud con cuatro cuerdas abiertas (H. Kawai, 1985).

Recientemente, Bern, Carrasco y Johanson (Z. Bern, 2008) (relación BCJ) han generalizado el resultado que se encontró utilizando teoría de cuerdas, la llamada relación KLT (Kawai-Lewellen-Tie) (H. Kawai, 1985). La comprensión de la doble copia está incluso a nivel de Lagrangianos (Johansson \& Nohle, 2017). La revolución de expresar teorías de gravedad como una doble copia cuántica incluso ha servido de ayuda para tener un nuevo formalismo que permite encontrar soluciones clásicas, por ejemplo en GR y Electrodinámica (Luna, 2015). En la siguiente sección se muestra un caso específico donde se calcula la autoenergía del gravitón como el cuadrado de la autoenergía del gluón.

\section{I Autoenergía del gravitón como doble copia cuántica}

Muchos físicos han estudiado los efectos cuánticos de la gravedad (Hawking, 1975). Uno de los cálculos mejor entendidos es la autoenergía del gravitón (Burns \& Pilaftsis, 2014), el cual es importante para conocer efectos cuánticos de la masa del gravitón. Básicamente se ha considerado el Lagrangiano de Hilbert-Einstein acoplado a materia, tanto escalar, fermiónica como vectorial. En este trabajo solo

\footnotetext{
${ }^{2}$ El gravitón es la partícula de spin-2, además es la partícula mediadora de la interacción gravitacional, tal como lo es el fotón para el caso de la electrodinámica cúantica.
} 
mostramos el caso del gravitón acoplado a una corriente fermiónica, por ejemplo una corriente de quarks, ver la referencia Burns and Pilaftsis (2014) para más detalles. Si calculamos la autoenergía del gravitón considerando un lazo fermiónico, la amplitud polarizada ${ }^{3}$ toma la siguiente forma [citar trabajo en progreso]

$$
\Pi_{\mathrm{G}}^{\mu v, \rho \sigma}(p)=F_{1}\left(p^{2}\right)\left(p^{\mu} p^{v} p^{\rho} p^{\sigma}+p^{4} \eta^{\mu v} \eta^{\rho \sigma}-p^{2}\left(\eta^{\mu v} p^{\rho} p^{\sigma}+\eta^{\rho \sigma} p^{\mu} p^{v}\right)\right),
$$

donde $F_{1}\left(p^{2}\right)$ es el factor de forma fermiónico que figura en el lazo (corrección cuántica) de la autoenergía del gravitón. Los autores de la referencia (Burns \& Pilaftsis, 2014) obtienen un resultado similar al de la ec. (22) utilizando el método tradicional de Feynman, en un trabajo de no menos de 20 páginas de cálculos. Sin embargo, podríamos tratar de reproducir la expresión tensorial de la ec. (22) si utilizamos el hecho que la gravedad a nivel de amplitudes (nivel perturbativo) se puede expresar como el cuadrado de una teoría cuántica (Z. Bern, 2008). Para lo que sigue, es necesario recordar la autoenergía del gluón ${ }^{4}$. La amplitud amputada para la autoenergía del gluón toma la siguiente forma (Peskin \& Schroeder, 1995)

$$
\mathcal{A}_{\text {gluon }}^{a b \mu v}=-g^{2}\left(\eta^{\mu v} p^{2}-p^{\mu} p^{v}\right) \delta^{a b} \Pi_{2}\left(p^{2}\right),
$$

donde $\Pi_{2}\left(p^{2}\right)$ es el factor de forma del lazo fermiónico en cromodinámica cuántica (QCD por sus siglas en inglés), ver el Apéndice 2. La delta de Kronecker $\delta^{a b}$ está asociada al álgebra del grupo de norma de QCD (Peskin \& Schroeder, 1995). Si se toma la parte que contiene la estructura tensorial $\left(\eta^{\mu v} p^{2}-p^{\mu} p^{v}\right)$ de la ecuación (23), y luego se eleva al cuadrado (cíclicamente en los indices $v, \rho \mathrm{y}$ $\sigma)$ obtenemos precisamente la parte tensorial de la amplitud que se muestra en la ecuación (22). Lo remarcable de poder expresar la autoenergía del gravitón como el cuadrado de la autoenergía del gluón, es el hecho que ambos resultados surgen de teorías completamente diferentes, es decir Lagrangianos manifiestamente diferentes y con diferentes simetrías (Carroll, 2004).

\section{I CONCLUSIONES}

En este trabajo hemos mencionado la importancia de la teoría del campo en la física moderna. La teoría de la relatividad general de Einstein describe con gran precisión los fenómenos clásicos del Cosmos. A nivel cuántico, nuestra mejor comprensión del Universo se debe al modelo estándar de partículas elementales. A pesar de que a nivel de Lagrangiano estas teorías no parecen tener ninguna conexión, cuando observamos a nivel de los bloques fundamentales, como lo son las amplitudes $\mathcal{A}$ surgen impresionantes relaciones matemáticas. Estas relaciones permiten ver teorías de gravedad como una doble copia de teorías cuánticas. Estos avances han permitido realizar una gran variedad de cálculos en gravedad cuántica que con métodos tradicionales eran imposibles. A nivel fundamental, nuevas relaciones matemáticas han surgido, lo que permite hacer un análisis de simetrías y dualidades que siguen siendo tema de investigación en la comunidad de amplitudes y fundamentos de teoría cuántica de campos.

\footnotetext{
${ }^{3}$ La amplitud polarizada es la amplitud amputada, sin considerar los gravitones en este caso, pero generalmente se refiere a no considerar las patas externas del diagrama de Feynman cuando estos son vectores de polarización.

4 el gluón es la partiícula mediadora de la interacción cuántica fuerte Peskin \& Schroeder 1995)
} 


\section{A | APÉNDICE}

\section{1। Factores de forma en Gravedad}

Las expresión análitica para el factor de forma a 1-lazo fermiónico en la autoenergía del gravitón es como sigue (Burns \& Pilaftsis, 2014)

$$
F_{1}\left(p^{2}\right)=\frac{\kappa^{2}}{1800(4 \pi)^{2}\left(p^{2}\right)^{2}}\left[\left(\alpha_{1}+\alpha_{4}\right) B_{0}\left(p^{2}, m_{H}^{2}, m_{H}^{2}\right)+\left(\alpha_{2}+\alpha_{5}\right) A_{0}\left(m_{H}^{2}\right)+\left(\alpha_{3}+\alpha_{6}\right)\right],
$$

con las funciones de Passarino-Veltman tomando la siguiente forma:

$$
\begin{gathered}
B_{0}\left(p^{2}, m_{1}^{2}, m_{2}^{2}\right) \equiv \quad(2 \pi \mu)^{4-d} \int \frac{d^{d} k}{i \pi^{2}} \frac{1}{k^{2}-m_{1}^{2}} \frac{1}{(k+p)^{2}-m_{2}^{2}} \\
=\frac{1}{\bar{\varepsilon}}+2-\ln \left(\frac{m_{1} m_{2}}{\mu^{2}}\right) \\
+\frac{1}{p^{2}}\left[\left(m_{2}^{2}-m_{1}^{2}\right) \ln \left(\frac{m_{1}}{m_{2}}\right)+\lambda^{1 / 2}\left(p^{2}, m_{1}^{2}, m_{2}^{2}\right) \cosh ^{-1}\left(\frac{m_{1}^{2}+m_{2}^{2}-p^{2}}{2 m_{1} m_{2}}\right)(25)\right. \\
A_{0}\left(m^{2}\right)=m^{2}\left(1+B_{0}\left(0, m^{2}, m^{2}\right)\right) .
\end{gathered}
$$

$\operatorname{con} \lambda(x, y, z) \equiv(x-y-z)^{2}-4 y z$. Además, las funciones $\alpha_{i}$ son las siguientes

$$
\begin{aligned}
& \alpha_{1}=-15\left[p^{2}-4 m_{\psi}^{2}\right]^{2}, \\
& \alpha_{2}=+30\left[8 m_{\psi}^{2}+p^{2}\right], \\
& \alpha_{3}=-16\left[15 m_{\psi}^{4}-10 m_{\psi} p^{2}+p^{4}\right], \\
& \alpha_{4}=-15\left[32 m_{\psi}^{4}+4 m_{\psi}^{2} p^{2}-3 p^{4}\right], \\
& \alpha_{5}=-30\left[3 p^{2}-16 m_{\psi}^{2}\right], \\
& \alpha_{6}=-480 m_{\psi}^{4}+20 m_{\psi}^{2} p^{2}+18 p^{4} .
\end{aligned}
$$

\section{2 | Factores de forma en Cromodinámica Cuántica}

Las expresión análitica para el factor de forma a 1-lazo fermiónico en la autoenergía del gluón es como sigue (Peskin \& Schroeder, 1995)

$$
\Pi_{2}\left(p^{2}\right)=\frac{T_{F}}{2 \pi^{2}} \int_{0}^{1} d x x(1-x)\left[\frac{2}{\varepsilon}+\ln \left(\frac{\tilde{\mu}^{2}}{m^{2}-p^{2} x(1-x)}\right)+O(\varepsilon)\right]
$$

\section{REFERENCIAS}

Burns, D., \& Pilaftsis, A. (2014, Diciembre). Matter quantum corrections to the graviton self-energy and the newtonian potential. Phys. Rev. D, 91, 27.

Carroll, S. M. (2004). Spacetime and Geometry, “An Introduction to General Relativity”. Pearson.

Cottingham, W. N., \& Greenwood, D. A. (2007). An introduction to the standard model of particle physics. Cambridge University Press.

DeWitt, B. S. (1967). Quantum theory of gravity. 3. applications of the covariant theory. Phys. Rev., 162, 18. 
Diaz-Cruz, J. L., \& Larios, B. O. (2016, Octubre). Stop decay with 1sp gravitino in the final state. Eur. Phys. J. C, $76(3), 12$.

Dirac, P. (1958). The principles of quantum mechanics. Oxford University.

Hawking, S. W. (1975). Particle creation by black holes. Commun. Math. Phys, 43, 22.

H. Kawai, S. H. H. T., D. C. Lewellen. (1985, Septiembre). A relation between tree amplitudes of closed and open strings. Nucl. Phys. B., 269, 23.

Jackson, D. (1998). Classical electrodynamics. Wiley; 3 edition.

J. Lorenzo Diaz-Cruz, M. A., Bryan Larios. (d). work in progress. $d, d(\mathrm{~d}), \mathrm{d}$.

J. Lorenzo Diaz-Cruz, O. M.-A., Bryan O. Larios. (2016, Agosto). An introduction to the massive helicity formalism with applications to the electroweak sm. J. Phys. Conf. Ser, 761(1), 8.

Johansson, H., \& Nohle, J. (2017). Conformal gravity from gauge theory. arXiv:1707.02965 [hep-th], 42.

Lancaster, T., \& Blundell, S. J. (2014). Quantum field theory for the gifted amateur. Oxford University Press.

Landau, L. D., \& Lifshitz, E. M. (1975). The classical theory of fields. Pergamon Press; 4th rev. English edition.

Larios, B. (2017). Helicity amplitudes for production of massive gravitino/goldstino. J.Phys.Conf.Ser., 912(1), 8.

Luna, A. (2015, Julio). The classical double copy for taub?nut spacetime. Phys. Lett. B, 750, 6.

Martin, S. P. (1998). A susy primer. Advances Series on High Energy Physics, 18, 1-98.

Nima Arkani-Hamed, J. K., Freddy Cachazo. (2010, Septiembre). The simplest quantum field theory. JHEP, 3, 89.

Penrose, R., \& MacCallum, M. A. (1972, Junio). Twistor theory: An approach to the quantization of fields and space-time. Phys. Rept, 6, 76.

Peskin, M. E., \& Schroeder, D. V. (1995). An introduction to quantum field theory. Westview Press; 1 edition.

Schwinger, J. S. (1951). On gauge invariance and vacuum polarization. Phys. Rev., 82, 16.

Weinberg, S. (1967, 11). A model of leptons. Phys. Rev. Let., 19, 1264-1266.

Weinberg, S. (1996). The quantum theory of fields, vol 1. Cambridge University Press.

Weinberg, S. (2003). The discovery of subatomic particles. Cambridge University Press.

Weinberg, S. (2013). Cosmology. Oxford University Press.

Witten, E. (2004). Perturbative gauge theory as a string theory in twistor space. Commun. Math. Phys., 252, 97.

Z. Bern, H. J., J. J. M. Carrasco. (2008, Mayo). New relations for gauge-theory amplitudes. Phys.Rev. D, $78(085011), 40$.

Zwiebach, B. (2004). An introduction to string theory. Cambridge University Press. 